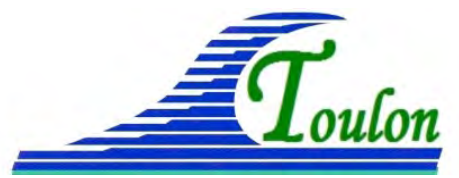

XIV èmes Journées Nationales Génie Côtier - Génie Civil

Toulon, 29 juin au $1^{\text {er }}$ juillet 2016

DOI:10.5150/jngcgc.2016.051 C Editions Paralia CFL

disponible en ligne - http://www.paralia.fr - available online

\title{
Instabilité post-sismique des digues. Une approche en déplacements
}

\author{
Jean François SERRATRICE ${ }^{1}$
}

1. Cerema, DTer Méditerranée, 30 rue Albert Einstein, CS 70499, 13593 Aix en Provence, France. jean-francois.serratrice@cerema.fr

\section{Résumé :}

La communication propose une récapitulation des observations des désordres produits dans les digues par des séismes, en vue de mieux connaître les mécanismes de déformation et de rupture de ces ouvrages. Puis, une méthode de calcul en déplacement est proposée, qui vise à décrire une cinématique de rupture post-sismique initiée par des pressions interstitielles. Le principe du calcul est exposé. Un exemple est commenté.

\section{Mots-clés :}

Digue, Remblai, Pression interstitielle, Séisme, Rupture, Méthode en déplacement.

\section{Introduction}

La préservation des digues se trouve à la croisée d'un double problème de risque, sismique et hydraulique et de ses conséquences en termes de gestion des risques, des secours ou de protection résiduelle, en plus des coûts des dommages. Un aperçu des cas de ruptures ou de tassements de remblais et de digues est donné, avant de présenter la méthode de calcul en déplacement. L'analyse se fonde sur la méthode des blocs. La rupture est déclenchée à la fin du séisme par les pressions interstitielles accumulées au cours des cycles dans le sol de fondation et la digue. Les déplacements cessent après remobilisation des forces stabilisatrices quand les pressions interstitielles sont dissipées. L'amplitude des déplacements et la durée du mouvement sont calculées. Un exemple est commenté pour montrer l'influence relative des principaux paramètres en jeu.

\section{Mécanismes de rupture des digues}

Les dommages provoqués à des remblais et des digues par les séismes dans différentes régions du monde ont fait l'objet d'analyses en retour. Chaque occurrence d'apparition de désordres sur un ouvrage en terre constitue bien souvent un cas particulier. Néanmoins, certaines tendances se répètent et des classifications des désordres provoqués par les séismes ont vu le jour, au Japon notamment (NAKAYAMA et al., 2007 ; SASAKI, 2009 ; OKHUBO et al., 2009 ; OKA et al. 2012). Ces retours d'expérience apportent une meilleure connaissance du comportement des ouvrages en terre. 
a)

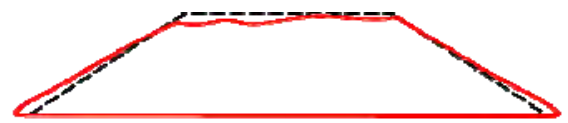

b)

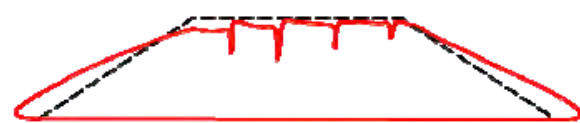

c)

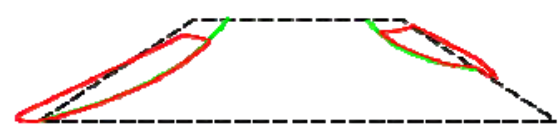

d)

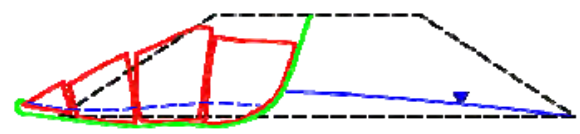

e)

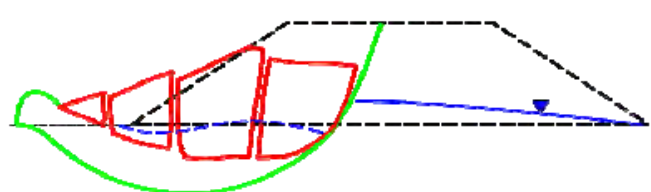

Figure 1. Récapitulation des classifications des ruptures de remblais sous séismes.

Remblais édifiés sur un massif horizontal. Ruptures dans leur plan transversal.

Une récapitulation des principaux modes de déformation des remblais sous l'effet des séismes est indiquée schématiquement sur la figure 1. En a) le tassement du remblai s'effectue sur lui-même. En b), les désordres affectent principalement le corps du remblai sans implication notable des sols de fondation. En c), des ruptures localisées affectent les flancs du remblai. En d), la liquéfaction de la couche basale du remblai ou d'une couche mince du sol de fondation, ou la dégradation de la résistance de ces terrains, produit la rupture du remblai de manière symétrique ou non. La rupture se manifeste par de larges crevasses longitudinales à regard amont qui séparent des blocs. Au centre du remblai, les déplacements sont quasi-verticaux. En e), le mécanisme de rupture affecte les sols de fondation en profondeur, suite à la liquéfaction des sols ou la dégradation de leur résistance. Le remblai est disloqué et s'étale. Dans cette classification, la méthode de calcul proposée plus bas se rapporte aux deux modes de rupture d) et e) de la figure 1.

\section{Méthodes de calcul}

\subsection{Méthode des blocs}

La "méthode des blocs" a été proposée par SARMA (1979) pour effectuer le calcul de la stabilité des pentes à l'équilibre limite. Les calculs sont réalisés à deux dimensions, dans un profil de la pente. La portion du massif comprise entre la surface de rupture et la surface libre est subdivisée en blocs rigides indéformables et de forme quelconque qui 


\section{XIV èmes Journées Nationales Génie Côtier - Génie Civil \\ Toulon, 29 juin au $1^{\text {er }}$ juillet 2016}

frottent les uns sur les autres, pour représenter un mécanisme de rupture approprié ou imposé par des discontinuités préexistantes.

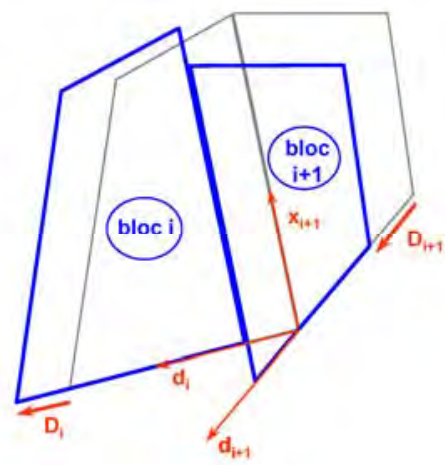

Figure 2. Mouvements relatifs de deux blocs en glissement sur leur base.

$D_{i}$ et $D_{i+1}$ sont les vecteurs mouvements des blocs.

Chaque discontinuité et chaque bloc peuvent posséder des propriétés particulières (poids volumique $\gamma$, cohésion c et angle de frottement $\phi$ ). En présence d'une nappe, les forces de pression d'eau sont calculées dans toutes les portions des discontinuités situées sous la nappe. Les efforts extérieurs éventuels sont ajoutés. La méthode des blocs tire son originalité de l'introduction d'une accélération horizontale $\mathrm{K}$ comme inconnue auxiliaire dans l'équilibre des blocs et dont une valeur critique $\mathrm{K}_{\mathrm{c}}$ à déterminer amène à l'équilibre limite. La méthode est directement applicable au calcul de l'équilibre pseudo-statique de l'ouvrage sous l'effet d'un séisme, en superposant un effort d'inertie horizontal et ou vertical au poids du volume instable, via des coefficients sismiques.

\subsection{Cinématique par la méthode des blocs}

L'assemblage de blocs constitué pour effectuer l'analyse pseudo-statique de la stabilité permet de représenter aussi une cinématique de la rupture. Les glissements des blocs sur leur base sont contraints par la forme de la surface de rupture. En se reportant à la figure 2, la condition de compatibilité des déplacements entre deux blocs attenants est donnée par l'expression vectorielle suivante (les deux blocs restent collés) :

$\mathrm{D}_{\mathrm{i}} \mathbf{d}_{\mathbf{i}}=\mathrm{D}_{\mathrm{i}+1} \mathbf{d}_{\mathbf{i}+\mathbf{1}}+\mathrm{X}_{\mathrm{i}+1} \mathbf{x}_{\mathbf{i}+\mathbf{1}}$

où $\mathbf{d}_{\mathbf{i}}$ est la direction de la base du bloc $\mathrm{i}, \mathbf{d}_{\mathbf{i}+\mathbf{1}}$ la direction de la base du bloc $\mathrm{i}+1, \mathbf{x}_{\mathbf{i}+\mathbf{1}}$ la direction de l'interface qui lie les blocs $i$ et $i+1$ (vecteurs unitaires) et $D_{i}, D_{i+1}, X_{i+1}$ les modules des déplacements des blocs sur leurs bases ou leur mouvement relatif. Par projection sur les directions $\mathbf{d}_{\mathbf{i}}$ et $\mathbf{d}_{\mathbf{i}+\mathbf{1}}$, un système peut être construit donnant $\mathrm{D}_{\mathbf{i + 1}}$ et $\mathrm{X}_{\mathbf{i}+1}$ comme solution, connaissant le module $\mathrm{D}_{\mathrm{i}}$ du déplacement du bloc $\mathrm{i}$. 


\section{Méthode de calcul}

\subsection{Principe de la méthode de calcul}

Une méthode est proposée ici pour analyser le mécanisme de rupture post-séisme d'un remblai ou d'une digue. L'instabilité résulte des pressions interstitielles accumulées pendant la durée du séisme le long de la surface de rupture potentielle. A cet instant initial du déclenchement, le déséquilibre mécanique se manifeste par une accélération imposée à la masse instable. Le déplacement de cette masse formée de plusieurs blocs est calculé en fonction du temps jusqu'à l'arrêt du mouvement quand les efforts statiques déstabilisateurs retrouvent un nouvel équilibre et/ou du fait de la dissipation des pressions interstitielles initiales. Le déplacement s'effectue à la fin ou après la fin du séisme, indépendamment des efforts sismiques d'inertie. Le déplacement final est comparé ensuite aux seuils préétablis dans le cadre d'une analyse performantielle de l'ouvrage en terre, voire comme scénario d'une analyse de risque.

\subsection{Equilibre initial et instabilité}

Une solution du problème de stabilité est recherchée pour commencer, parmi d'autres, par la méthode des blocs (surface de rupture, découpage en blocs, identification des propriétés mécaniques des discontinuités, nappe, efforts extérieurs, etc.). Ces données ne sont pas actualisées dans la suite du calcul. Les résistances au cisaillement sont définies en termes de contraintes effectives. Une distribution de surpressions interstitielles $\Delta \mathrm{u}_{0}$ est donnée le long de la surface de rupture. Elles représentent les pressions interstitielles accumulées pendant le séisme. Elles sont définies à la base de chacun des blocs $\mathrm{i}$ en fonction des contraintes normales effectives $\sigma_{0 \mathrm{i}}^{\prime}$, calculées à l'étape précédente, par un coefficient $r_{u 0}$, ou taux d'accumulation des pressions interstitielles, qui est unique pour l'ensemble de la surface de rupture. Pour le bloc i :

$\Delta \mathrm{u}_{0 \mathrm{i}}=\mathrm{r}_{\mathrm{u} 0} \sigma_{0 \mathrm{i}}^{\prime}$

Le déséquilibre mécanique ainsi créé se manifeste par une accélération imposée à chacun des blocs. Le calcul du déplacement de la masse instable commence à cet instant initial du déclenchement. Pendant le mouvement des blocs, la dissipation des surpressions interstitielles le long de la surface de rupture s'effectue en référence à la théorie de la consolidation unidimensionnelle des sols. Un temps caractéristique $t_{d}$ contrôle cette dissipation en lien avec le facteur temps $T_{v}=t / t_{d}$, où $t$ est le temps.

\subsection{Déroulement du calcul}

Le calcul des déplacements des blocs s'effectue pas à pas en fonction du temps à partir du déclenchement initial provoqué par les surpressions interstitielles. Pour chaque bloc et pendant chacun des pas de temps, l'incrément de vitesse et l'incrément de déplacement sont calculés par double intégration, connaissant l'accélération du bloc au 


\section{XIV ${ }^{\text {èmes }}$ Journées Nationales Génie Côtier - Génie Civil \\ Toulon, 29 juin au $1^{\text {er }}$ juillet 2016}

temps t qui résulte du déséquilibre des forces de cisaillement sur sa base. L'incrément de dissipation des pressions interstitielles est calculé sous le bloc. Puis la règle (1) de compatibilité des vitesses et des déplacements est appliquée le long de la surface de rupture à partir du bloc de pied de façon à rendre continu le mouvement d'ensemble. Le calcul s'arrête par un effet géométrique ou l'effet de la restauration des forces de cisaillement résistantes ou les deux effets simultanément. Dans le premier cas, les efforts statiques déstabilisateurs retrouvent un équilibre. Dans le second cas, les forces résistantes sont rétablies après dissipation des surpressions interstitielles. Le résultat final s'exprime par le déplacement total de chacun des blocs depuis l'initiation de l'instabilité, puis par le temps de parcours. Un logiciel spécifique a été mis au point.

(a)

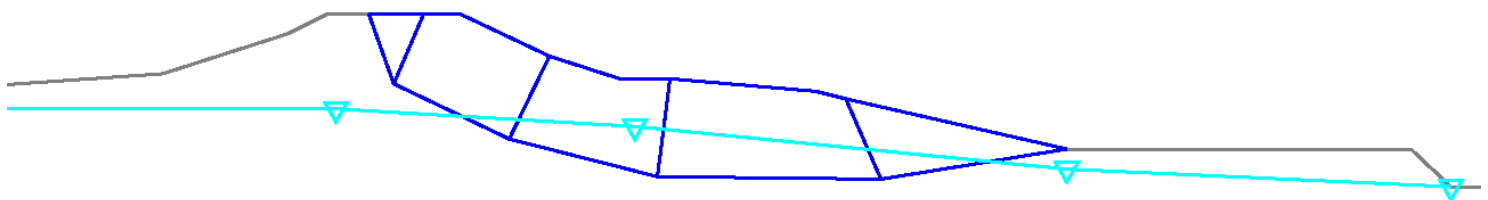

(b)

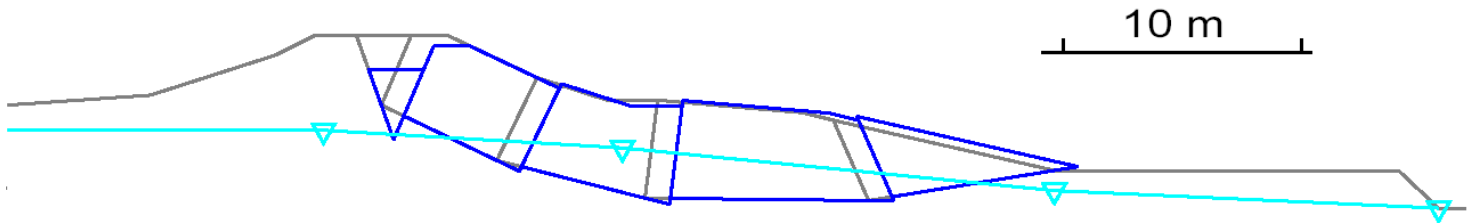

Figure 3. Profil de calcul d'une digue de la rivière Naruse au Japon.

a) Discrétisation du profil en cinq blocs. b) Cinématique de rupture.

\section{Exemple}

\subsection{Présentation}

En 2004, le séisme Niyagi-ken Hokubu a provoqué de sérieux dommages sur les digues de la rivière Naruse. L'observation des désordres a fait l'objet d'une analyse postsismique par NAKAYAMA et al. (2007). Comme de nombreux ouvrages, la digue est hétérogène et a été rehaussée et élargie au fil du temps. Dans cette section de l'ouvrage, l'ancienne digue est constituée par des sols cohérents et les nouveaux matériaux rapportés sont sableux. La liquéfaction des terrains sableux de fondation de la digue et ceux qui constituent la région basale du corps du remblai explique la rupture. Des crevasses longitudinales larges et profondes séparent des blocs du remblai initial.

En vue du calcul dédié à cet exemple, la masse instable est discrétisée en cinq blocs dans le profil de la digue, comme indiquée sur la figure 3a, d'après le schéma de rupture. 


\section{Thème 4 - Ouvrages portuaires, offshore et de plaisance}

Le calcul pseudo-statique effectué à l'équilibre limite par la méthode des blocs, tenant compte des propriétés mécaniques des sols proposées par les auteurs, aboutit à une accélération horizontale déstabilisatrice qui est égale à $4,4 \mathrm{~m} / \mathrm{s}^{2}$. En comparaison, les accélérations estimées sur le site sont comprises entre 3 et $5,5 \mathrm{~m} / \mathrm{s}^{2}$ pendant les secousses sismiques.

Tableau 1. Jeux de paramètres pour les calculs paramétriques.

\begin{tabular}{lllll}
\hline Paramètres & Jeux des paramètres \\
\hline angle de frottement des discontinuités $\phi\left(^{\circ}\right)$ & 28 & 30 & 32 & 34 \\
taux d'accumulation des pressions interstitielles $r_{u}()$ & 0,70 & 0,80 & 0,90 & 0,95 \\
temps caractéristique de dissipation $t_{d}(\mathrm{~s})$ & 0,5 & 1 & 2,5 & 5 \\
\hline
\end{tabular}

\subsection{Résultats du calcul des déplacements}

Les calculs sont effectués en contraintes effectives dans la modélisation proposée ici. En l'absence de données des caractéristiques de résistance des sols dans ces conditions, les propriétés suivantes ont été adoptées, qui sont reportées dans le tableau 1. Elles sont communes à tous les blocs.

Les figures 4, 5 et 6 montrent les résultats des calculs, avec le déplacement du bloc de pied et la dissipation des pressions interstitielles sous le bloc de pied en fonction du temps. L'influence de $\phi$ est grande. L'amplitude du déplacement diminue quand l'angle de frottement $\phi$ augmente. Concernant le coefficient $r_{u} 0$, les déplacements et les temps de parcours sont plus grands quand les surpressions interstitielles initiales sont plus grandes, en liaison avec les accélérations plus grandes qui provoquent le déclenchement de la rupture. L'influence du temps caractéristique de dissipation des pressions interstitielles $t_{d}$ est forte. Les pressions interstitielles initiales $\left(r_{u 0}\right)$ puis les conditions de dissipation de ces pressions $\left(t_{d}\right)$ sont déterminantes dans le déclenchement et le développement du mouvement. La non dissipation des pressions interstitielles conduit à des ruptures catastrophiques, y compris pour les faibles pentes, ce qui est bien connu et vérifié par l'expérience.

Les calculs paramétriques et les courbes des figures 4,5 et 6 aboutissent à des déplacements du bloc de pied très variables et fortement dépendants des paramètres considérés. Ils dépassent 1,5 m ici pour des temps de parcours de quelques secondes. La figure 3b montre le profil cinématique calculé pour un déplacement de $1 \mathrm{~m}$ du bloc de pied. Les déplacements des blocs vont croissants en allant du pied vers la crête du glissement, d'après la règle de compatibilité (1). Ici, le déplacement du bloc 5 en crête vaut $1,5 \mathrm{~m}$ sur sa base qui est très redressée, avec une composante verticale de plus de 1,4 m. Ce mécanisme explique les forts "tassements" souvent observés en crête de remblai, néfastes à l'intégrité des digues du fait de la perte de revanche hydraulique et la fissuration produite en profondeur dans le corps du remblai (figure 3b). 


\section{XIV èmes Journées Nationales Génie Côtier - Génie Civil \\ Toulon, 29 juin au $1^{\text {er }}$ juillet 2016}
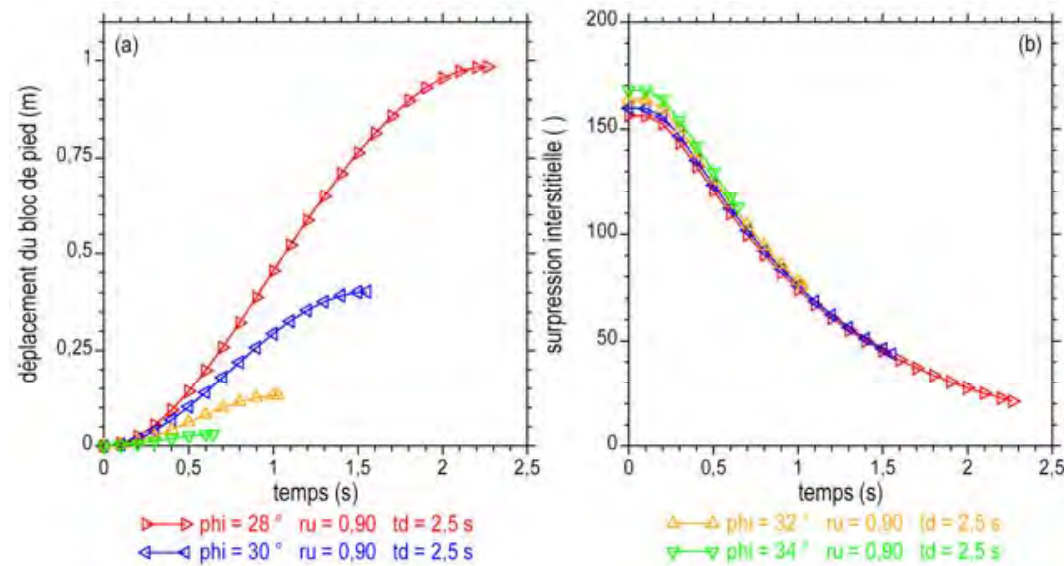

Figure 4. Influence de l'angle de frottement $\phi$.

a) Déplacement du bloc de pied. b) Dissipation de la surpression interstitielle.

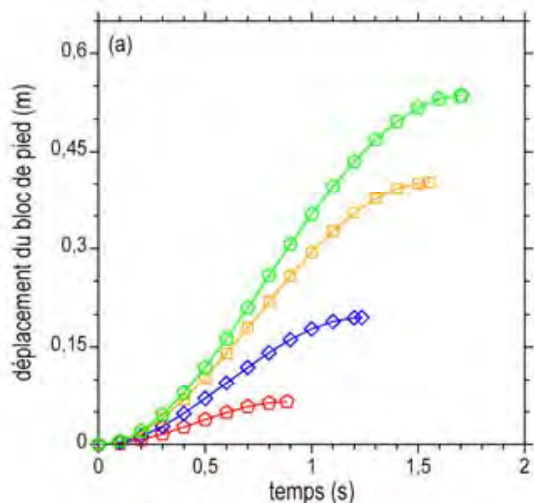

$\triangle \mathrm{phi}=30^{\circ} \mathrm{ru}=0,70 \mathrm{td}=2,5 \mathrm{~s}$ $\diamond \mathrm{phi}=30^{\circ} \mathrm{ru}=0,80 \mathrm{td}=2,5 \mathrm{~s}$

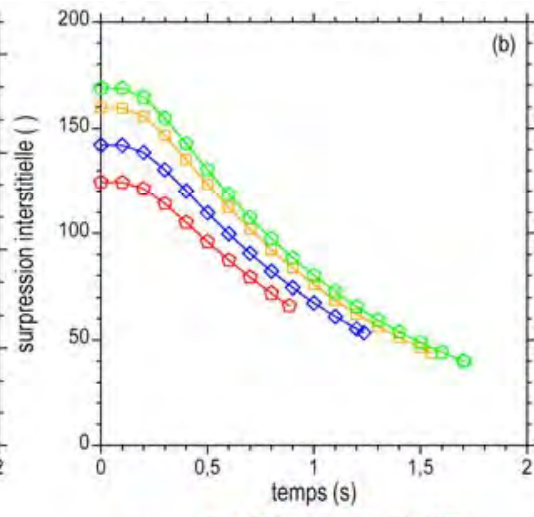

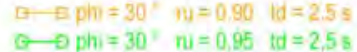

Figure 5. Influence du coefficient de pression interstitielle initiale $r_{u 0}$.

a) Déplacement du bloc de pied. b) Dissipation de la surpression interstitielle.
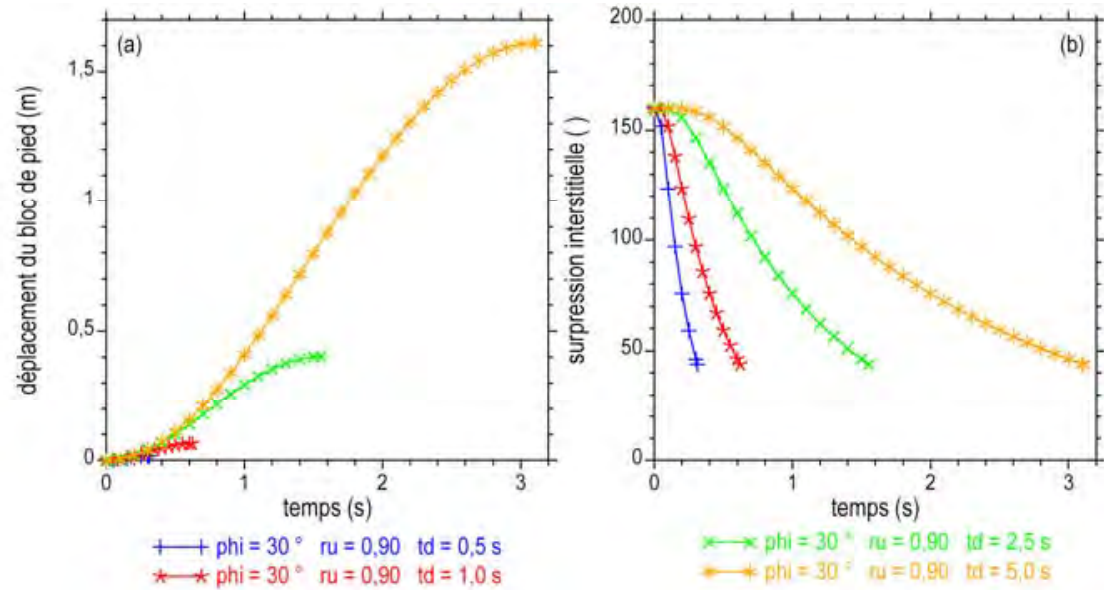

Figure 6. Influence du temps caractéristique de dissipation $t_{d}$.

a) Déplacement du bloc de pied. b) Dissipation de la surpression interstitielle. 


\section{Conclusions}

Dans la méthode proposée, le calcul de la stabilité initiale du massif et du remblai avant séisme est effectué par la méthode des blocs en mode pseudo-statique. Puis, des pressions interstitielles accumulées au cours du séisme sont distribuées le long de la surface de rupture et elles déterminent l'instabilité. La méthode des blocs est utilisée pour calculer la cinématique des déplacements post-sismiques, jusqu'au rétablissement de l'équilibre. Des calculs paramétriques ont été effectués sur la base d'un exemple de rupture d'une digue au Japon, suite à la liquéfaction des sols sableux en présence.

Les hypothèses simplificatrices qui ont été admises réduisent à trois le nombre des paramètres du problème. D'après l'exemple étudié, l'influence de l'angle de frottement est grande. L'amplitude des pressions interstitielles initiales puis les conditions de dissipation de ces pressions sont déterminantes dans le déclenchement et le développement du mouvement. La non-dissipation des pressions interstitielles conduit à des ruptures catastrophiques.

La méthode de calcul en déplacement présentée ici ne constitue qu'une étape dans le processus de calcul de la stabilité d'une digue. Malgré sa simplicité et les hypothèses fortes qui la marquent, sa mise en œuvre fait appel en amont à des paramètres qui sont discriminants, comme indiqué par les calculs paramétriques de l'exemple, et qui nécessitent d'être identifiés avec soin. En aval, les déplacements calculés sont à comparer aux niveaux de dommage acceptables définis en classes de performances, sur la base d'une échelle des déplacements admissibles par l'ouvrage en terre.

\section{Références bibliographiques}

NAKAYAMA O., SASAKI Y., SEKISAWA M., HIRATSUKA T., SUZUKI Y. (2007). Deformation of a river dike due to the Miyagi-Ken Hokubu earthquake. Proc. 4th Int. Conf. on Earthquake Geotechnical Engng., paper n 1240, 12 p.

OKHUBO K, FUJIOKA K., YASUDA S. (2009). Slump failure of highway embankments during the 2004 Niigataken Chuetsu earthquake. Proc. Earthquake geotechnical case histories for performance-based design, CRC Press/Balkema, pp 71-82. http://dx.doi.org/10.1201/NOE0415804844.ch4

OKA F., TSAI P., KIMOTO S., KATO R. (2012). Damage patterns of river embankments due to the 2011 off the Pacific Coast of Tohoku Earthquake and a numerical modeling of the deformation of river embankments with a clayey subsoil layer. Soils and Foundations, vol. 52(5), pp 890-909. http://dx.doi.org/10.1016/j.sandf.2012.11.010 SARMA S.K. (1979). Stability analysis of embankments and slopes. J. Soil Mech. and Found. Div., ASCE, Vol. 105(12), pp 1511-1523.

SASAKI Y. (2009). River dike failures during the 1993 Kushiro-oki earthquake and the 2003 Tokachi-oki earthquake. Proc. Earthquake geotechnical case histories for performance-based design, CRC Press/Balkema, pp 131-157. 